\title{
INFLUENCIA DE LA APARIENCIA DE LAS MAMAS \\ EN LA CALIDAD DE VIDA. DIFERENCIAS ENTRE POBLACIÓN PREOPERATORIA DE MAMOPLASTÍA DE REDUCCIÓN Y POBLACIÓN CONTROL*
}

\author{
Drs. Stefan Danilla E. ${ }^{1,2}$, Pedro Cuevas T. ${ }^{3}$, Ma. Elsa Calderón G. ${ }^{3}$, \\ Als. José Ignacio Vergara O. ${ }^{3}$, Felipe Soto V. ${ }^{3}$, Drs. Cristian Erazo C..$^{1,2}$, \\ Marco Ríos V. ${ }^{3}$, Als Alma Cruz S. ${ }^{3}$, Sofía Serra R. ${ }^{3}$, Paula Silva R. ${ }^{3}$

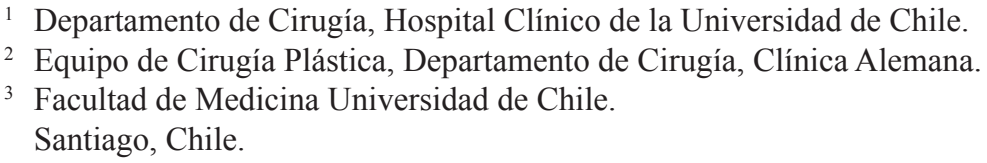
Susana Benítez S. ${ }^{1}$, Patricio Andrades C. ${ }^{1}$, Rolando Schulz R. ${ }^{1}$, Sergio Sepúlveda P. ${ }^{1,2}$,

\section{Abstract \\ Criterion validity of a questionnaire to assess quality of life associated to the appearance of breasts in women}

Background: The Breast QoL Reduction and Mastopexy Module assesses quality of life associated to breast appearance from the point of view of the patient. Its Spanish version was recently validated in Chile. Aim: To assess the criterion validity of the instrument applying it in different populations of women and in the pre and post-operative periods. Material and Methods: The questionnaire was applied to six women subjected to reduction mammoplasty in the pre and post-operative period. It was also applied to 20 women hospitalized for other causes and 20 female medical students. Results: There were significant differences between operated women and their non-operated counterparts in the domains body self-image, social performance and physical symptoms. No differences in sexuality were found. However this last question was answered only by four operated patients, since two declared not to have sex life. Conclusions: The questionnaire is useful to assess quality of life associated to the appearance of breasts.

Key words: Mammoplasty, quality of life, breasts.

\section{Resumen}

Objetivo: El Breast Q Reduction and Mastopexy Module es un instrumento específico para la evaluación de calidad de vida asociado a mamas desde la perspectiva del paciente, recientemente validado para uso en español en Chile. Cuenta con evaluación pre y postoperatoria. El objetivo del presente estudio es conocer

*Recibido el 10 de junio de 2013 y aceptado para publicación el 2 de septiembre de 2013.

Ninguno de los autores tiene conflictos de interés potencialmente relevantes al estudio realizado.

Correspondencia: Dr. Stefan Danilla E.

Santos Dumont No 999, Santiago, Chile.

drstefandanilla@gmail.com 
la validez de criterio al comparar las distribuciones en distintas poblaciones de individuos así como la diferencia del puntaje entre el pre y postoperatorio. Materiales y Métodos: El instrumento validado en español Breast $Q$ Reduction-Mastopexy Module fue sometido a prueba para evaluar su validez de criterio comparando los resultados de la escala en pacientes que deseaban someterse a cirugía versus 2 grupos control, uno de pacientes hospitalizados por otra causa y otro de estudiantes de medicina. Resultados: Se aplicó la encuesta a 46 mujeres, 6 pacientes preoperatorios de mamoplastía de reducción, 20 alumnas de medicina y 20 pacientes hospitalizadas por otra causa. Hubo diferencias estadísticamente significativas entre las pacientes y los grupos control en los dominios autoimagen corporal $(\mathrm{p}=0,0001)$, desempeño social $(\mathrm{p}=0,0035)$ y sintomas fisicos $(\mathrm{p}=0,0058)$, no se observaron diferencias en el dominio de sexualidad $(\mathrm{p}=0,1432)$, sin embargo, sólo 4/6 pacientes contestaron la encuesta ya que las otras 2 declararon no tener vida sexual. Conclusiones: Estos resultados preliminares sugieren que la versión en español del Breast Q Reduction-Mastopexy Module es un instrumento útil para evaluar calidad de vida asociada a las mamas en mujeres chilenas.

Palabras clave: Mama, mamoplastía de reducción, reducción mamaria, calidad de vida, medicina basada en evidencia, instrumentos de medición, PROs.

\section{Introducción}

La cirugía mamaria constituye uno de los motivos más frecuentes de consulta en cirugía plástica. En las últimas décadas ha existido un continuo desarrollo en las técnicas quirúrgicas, de imagen y en el soporte tecnológico que han permitido ofrecer resultados quirúrgicos más confiables. No obstante lo anterior, hasta hace poco no existían instrumentos que permitieran evaluar de forma objetiva y comparable los resultados en cirugía plástica. Con el fin de poder estandarizar la evaluación de resultados se han diseñado instrumentos que permiten evaluar de manera cuantitativa los resultados obtenidos. Uno de ellos es el Breast Q Reduction and Mastopexy, (Breast-Q) recientemente validado para uso en Chile por nuestro grupo ${ }^{1}$. El objetivo del presente estudio es evaluar la validez de criterio del Breast$Q$, realizando una comparación del puntaje obtenido por los pacientes programados para realizarse una mamoplastía de reducción con dos grupos control y además evaluar la diferencia entre el puntaje pre y post operatorio de los pacientes operados.

\section{Material y Método}

\section{Diseño}

Para lograr nuestro objetivo diseñamos un estudio observacional prospectivo, llevado a cabo en un hospital universitario. El estudio incluye 46 pacientes distribuidos en un grupo activo de 6 pacientes en su preoperatorio de mamoplastía de reducción y dos grupos control formado por 20 estudiantes de medicina y 20 pacientes hospitalizados por otras patologías.

\section{Mediciones}

Las variables a evaluar fueron el grado de satisfacción con la apariencia mamaria en pacientes que serán sometidas a una reducción mamaria y compararlas con la del grupo control utilizando la versión validada en español del Breast $Q$ Reduction and Mastopexy Module con el fin de poder evaluar la validez de criterio del instrumento. La validez de constructo y propiedades psicométricas de la escala ya han sido publicadas por sus autores ${ }^{2}$. La encuesta incluye 4 dominios divididos en 39 ítems pertenecientes a dominios autoimagen corporal, desempeño social, sexualidad y síntomas físicos; administrados en preguntas tipo Likert de 5 puntos $^{3}$, con un rango de puntaje entre 39-184 puntos.

\section{Estadística}

La diferencia de puntaje entre grupos se evaluó con la prueba de Kruskall-Wallis, la diferencia en el grupo de mamoplastía reductiva entre el pre y postoperatorio se evaluó con la prueba de Wilcoxon. Las variables continuas entre grupos se evaluaron con la prueba de ANOVA y la prueba de $\chi^{2}$ para las variables categóricas. La confiabilidad se midió con el Alfa de Cronbach. Para todas las pruebas se utilizó un nivel alfa de 5\%, en el análisis de los datos se utilizó el software Stata 10.2® (Stata Corp, TX, EEUU).

\section{Resultados}

Entre enero y mayo de 2011 se reclutaron 46 pacientes. En la Tabla 1 se detallan las características generales de los pacientes de cada grupo. La confiabilidad de la escala evaluada con el alfa de Cronbach alcanzó el 97,01\%. La mediana de respuesta por dominio en los distintos grupos se presenta en la Tabla 2. Se aprecian diferencias estadísticamente significativas entre las pacientes y los grupos control en los dominios autoimagen corporal ( $\mathrm{p}=0,0001)$, desempeño social $(\mathrm{p}=0,0034)$ y sintomas físicos $(\mathrm{p}=0,0058)$, no se observaron diferencias en el 
Tabla 1. Características generales de los pacientes por grupo expresados como promedio \pm desviación estándar

\begin{tabular}{|lccc|}
\hline & Grupo activo & Control 1 Hospitalizado & Control 2 Estudiantes \\
n pacientes & 6 & 20 & 20 \\
Edad (años) & $29 \pm 16,02$ & $51,5 \pm 15$ & $22,6 \pm 2$ \\
Escolaridad (años) & $13,5 \pm 2,58$ & $11,7 \pm 4,7$ & $16 \pm 0,9$ \\
IMC $\left(\mathrm{kg} / \mathrm{m}^{2}\right)$ & $25 \pm 0,54$ & $25,2 \pm 1,6$ & $22,9 \pm 2,4$ \\
\hline
\end{tabular}

Tabla 2. Mediana de Breast-Q® score por grupo y dominio

\begin{tabular}{|llcccc|}
\hline Dominio & & Estudiantes & Hospitalizadas & Operadas & P value \\
Autoimagen & 44 puntos & 37,5 & 32,5 & 16 & 0,001 \\
Desempeño social & 45 puntos & 37 & 35,5 & 21 & 0,0034 \\
Sexualidad & 25 puntos & 19,5 & 20,5 & 12 & 0,1411 \\
Síntomas físicos & 70 puntos & 24 & 25 & 47 & 0,0058 \\
\hline
\end{tabular}

Tabla 3. Porcentaje del máximo teórico pre y postoperatorio

\begin{tabular}{|lccc|}
\hline Dominio & Evaluación preoperatoria & Evaluación postoperatoria & p value \\
\hline Autoimagen corporal & $35,6 \%$ & $60,5 \%$ & 0,2366 \\
Desempeño social & $44,8 \%$ & $62,9 \%$ & 0,3309 \\
Sexualidad & $36 \%$ & $41,3 \%$ & 0,7040 \\
Síntomas físicos & $33,0 \%$ & $80 \%$ & 0,0056 \\
\hline
\end{tabular}

dominio de sexualidad ( $\mathrm{p}=0,1432)$, sin embargo, sólo 4 de 6 pacientes contestaron la encuesta ya que 2 de ellas no tenían vida sexual. En la Tabla 3 se puede observar el porcentaje del máximo teórico de puntaje por dominio en el pre y postoperatorio de las pacientes sometidas a mamoplastía de reducción. Se observa que en todos los dominios evaluados hubo mejoría, alcanzando esta misma, un valor estadísticamente significativo para el dominio de síntomas físicos. Nuevamente en el dominio de sexualidad se aprecia la menor variación, pero su resultado se ve limitado por las razones antes mencionadas.

\section{Conclusiones}

La creación, desarrollo y aplicación de instrumentos objetivos de evaluación de resultados desde la perspectiva del paciente constituye un campo en amplio desarrollo y de gran trascendencia actual en la investigación médica. El poder evaluar de forma objetiva y reproducible el grado de satisfacción de una cirugía por parte del paciente ha adquirido cada vez mayor trascendencia ${ }^{4}$, tanto del punto de vista científico al permitir objetivar y cuantificar los resultados con una u otra técnica quirúrgica, desarrollar estudios epidemiológicos; como también desde el punto de vista de la relación médico-paciente, al poder demostrar con parámetros objetivos los cambios y mejorías 5 .

En cirugía reconstructiva, resulta muy relevante el poder "cuantificar" los cambios obtenidos entre el pre y el posoperatorio ya que esto nos permite reforzar la sensación de mejoría en nuestros pacientes ${ }^{4}$.

Los resultados de nuestro estudio señalan que el instrumento Breast $Q$ Reduction and Mastopexy, (Breast- $Q$ ) validado en español posee una adecuada validez de criterio, transformándose en una herramienta útil en la evaluación del resultado de la cirugía de reducción mamaria. Por lo anterior confiamos en que su uso se torne rutinario en la evaluación de los pacientes sometidos a mamoplastía reductiva, ya que su uso adecuado permitirá la comparación de resultados entre pacientes y técnicas; permitiendo la realización de estudios de evaluación de resultado y estudios comparativos que sin lugar a dudas contribuirán al avance científico en cirugía plástica. 


\section{Referencias}

1. Cuevas P, Calderón M, Erazo C, Benítez S, Andrades P, Sepúlveda S, y col. Mamoplastía de reducción: resultados desde la perspectiva del paciente. Validación lingüística y psicométrica del Breast Q Reduction and Mastopexy Module Instrument. Rev Chil Cir. 2013;65:146-9.

2. Pusic A, Klassen A, Scott A, Klok J, Cordeiro P, Cano S. Development of a new patient reported outcome measure for breast surgery: The BREAST-Q. Plast Reconstr
Surg. 2009;124:345-53.

3. Likert R. A Technique for the Measurement of Attitudes". Archives of Psychology 1932;140:44-53.

4. Cano SJ, Browne JP, Lamping DL. Patient-based measures of outcome in plastic surgery: Current approaches and future directions. Br J Plast Surg. 2004;57:1-11.

5. Patel KM, Hannan CM, Gatti ME, Nahabedian MY. A head to-head comparison of quality of life and aesthetic outcomes following immediate, staged-immediate, and delayed oncoplastic reduction mammaplasty. Plast Reconstr Surg. 2011;127:2167-75. 